\title{
Erratum to: An MR-Conditional High-Torque Pneumatic Stepper Motor for MRI-Guided and Robot-Assisted Intervention
}

\author{
Yue Chen, ${ }^{1}$ KA-Wai Kwok, ${ }^{1,2,3}$ and Zion Tsz Ho Tse ${ }^{1}$
}

${ }^{1}$ College of Engineering, The University of Georgia, Athens, GA, USA; ${ }^{2}$ Brigham and Women's Hospital, Harvard Medical School, Boston, MA, USA; and ${ }^{3}$ Department of Mechanical Engineering, The University of Hong Kong, Hong Kong, China

Erratum to: Annals of Biomedical Engineering (2014)

\section{2(9): 1823-1833}

DOI 10.1007/s10439-014-1049-x

This erratum is to correct for the motor power value in Fig. 8. The ranking of the proposed motor and the motor developed by Masamune et al. in terms of the power performance index is shifted. In addition, the following sentence ("The power of our presented motor is similar to that of one designed by Stoianovici et al., both of them far overweigh the other two motors.") in page 8 needs to be removed. The major contributions and findings of this paper are not affected by this error and the corrected version of Fig. 8 is shown here.

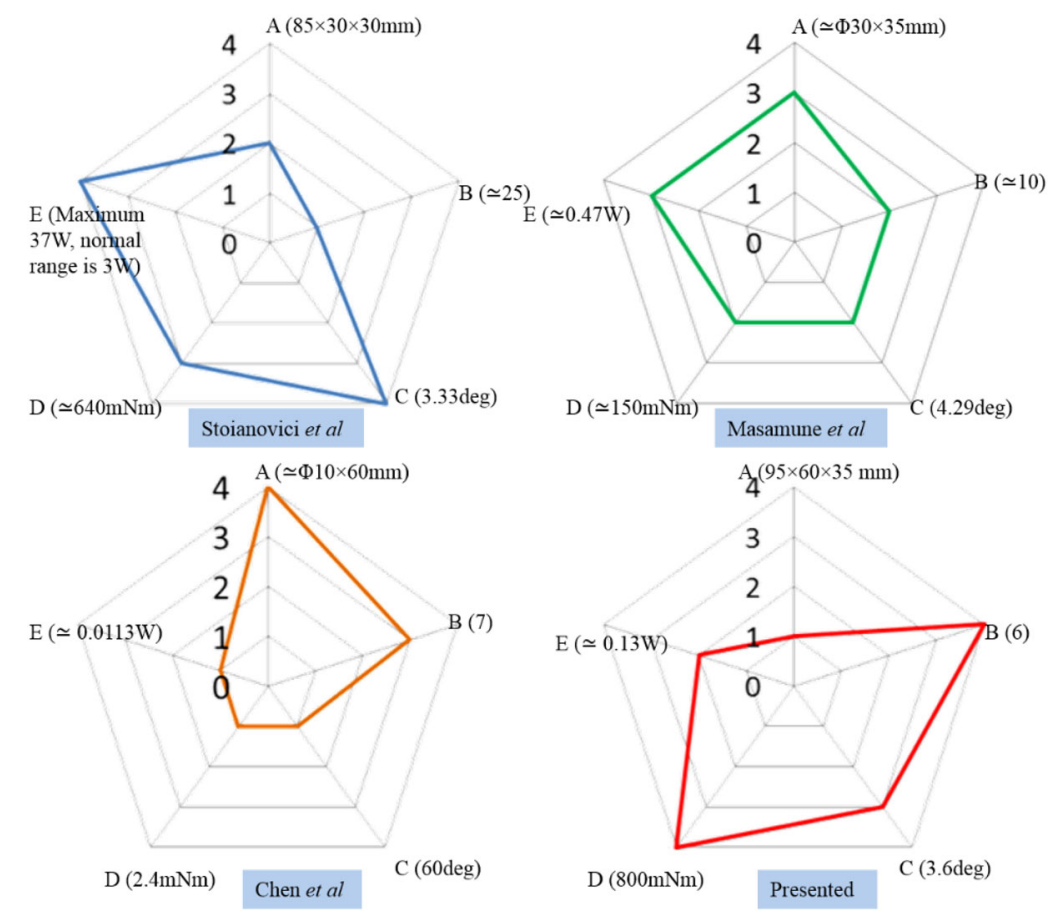

FIGURE 8. Comparison among five evaluation standards. Notes that rank 4 denotes the best in its corresponding standard. The larger the size of pentagon, the better is the overall performance in terms of A: motor dimension, B: number of components, C: step size, D: motor output torque, E: power.

Address correspondence to Ka-Wai Kwok, Department of Mechanical Engineering, The University of Hong Kong, Hong Kong, China. Electronic mail: kwokkw@hku.edu

The online version of the original article can be found under doi: 10.1007/s10439-014-1049-x. 